\title{
Esophagojejunostomy through minilaparotomy after laparoscopic total gastrectomy
}

\author{
Hiroshi Okabe, Seiji Satoh, Harutaka Inoue, Masato Kondo, Jun-ichiro Kawamura, Akinari Nomura, \\ Satoshi Nagayama, Suguru Hasegawa, Atsushi Itami, Go Watanabe, and Yoshiharu Sakai \\ Department of Surgery, Kyoto University Graduate School of Medicine, 54 Kawahara-cho, Shogoin, Sakyo-ku, Kyoto 606-8507, Japan
}

\begin{abstract}
Although laparoscopic distal gastrectomy (LDG) has been accepted as a surgical option for the treatment of early gastric cancer, laparoscopic total gastrectomy (LTG) has been adopted less often, because a more difficult surgical technique is required for reconstruction. To reduce the technical difficulties, we made some modifications to the functional end-to-end anastomosis technique and performed esophagojejunal anastomosis through a minilaparotomy. First, for easier handling of the esophagus, the first application of the linear stapler to create the esophagojejunal anastomosis was performed before transection of the esophagus. Second, the jejunal limb was anastomosed to the left side of the esophagus, which, compared with the right side, made available more free space, sufficient to operate the stapling device. Third, to close the entry hole and complete the gastrectomy concurrently, a linear stapler was applied through the left lower trocar. With this technique, the closure of the access opening was performed easily and was monitored directly through the minilaparotomy. We successfully performed LTG with Roux-en-Y reconstruction using our modified procedure in seven patients without any anastomotic complications. We believe our procedure is a secure and reliable method for reconstruction after LTG and will facilitate adoption of LTG as a surgical option for patients with early upper gastric cancers.
\end{abstract}

Key words Gastric cancer · Laparoscopic surgery · Total gastrectomy $\cdot$ Esophagojejunostomy

\section{Introduction}

Laparoscopic distal gastrectomy (LDG) is becoming accepted in several countries as one of the safe and feasible surgical modalities for early gastric cancer, although the long-term outcome awaits confirmation in large-scale randomized control trials $[1,2]$. However, laparoscopic total gastrectomy (LTG) for gastric cancer

Offprint requests to: $\mathrm{H}$. Okabe

Received: March 29, 2007 / Accepted: July 13, 2007 has not become as popular as LDG. The more difficult surgical technique of reconstruction after LTG compared with LDG, especially for esophagojejunostomy, appears to prevent the widespread acceptance of LTG. This article describes our procedure of extracorporeal Roux-en-Y reconstruction after LTG, which can be completed securely through a 5-cm minilaparotomy. In this procedure, by modifying a functional end-to-end anastomosis technique, esophagojejunal anastomosis is successfully created without hand sewing.

\section{Patients and methods}

\section{Patients}

Between November 2005 and August 2006, seven patients (two male and five female) with gastric cancer underwent LTG at our institution. These patients had a median age of 62 years (range, 32 to 82 years) and a body mass index of 20.3 (range, 17.0 to 24.0). All patients were diagnosed with gastric cancer, which was located in the upper third of the stomach and the depth of tumor invasion was limited to the proper muscular layer without lymph node involvement or invasion to the esophagus. These diagnoses were based on preoperative examinations, including gastrointestinal endoscopy, upper gastrointestinal series, and abdominal computed tomography (CT) scan.

\section{Surgical technique}

The patient was placed in a modified lithotomy position. The surgeon stood on the right side of the patient, with the first assistant on the left, and the endoscopist standing between the patient's legs. After pneumoperitoneum was established using the open technique through the umbilicus, four operating ports (Fig. 1B, C, D, and E) were placed in the upper abdomen. The left lobe of 


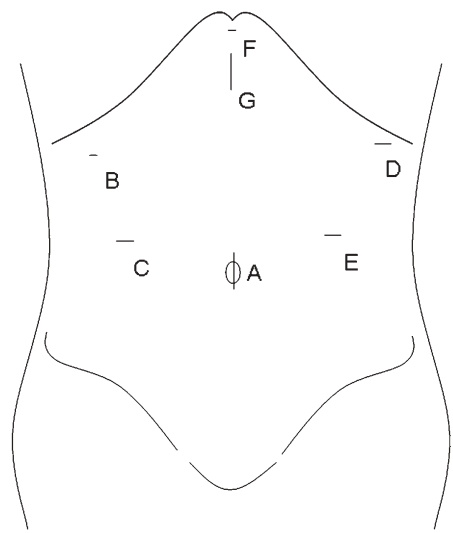

Fig. 1. Placement of the surgical ports; $12-\mathrm{mm}$ ports were used for ports $A, C, D$, and $E$. A 5-mm port was used for $B$. A videolaparoscope was introduced through port $A$. A liver retractor was introduced from $F$. A 5-cm minilaparotomy for reconstruction was made as $G$

the liver was retracted using a liver retractor and the round ligament was tied to the abdominal wall using 2-0 silk with an Endoclose suture carrier (Tyco Healthcare Japan, Tokyo, Japan,) to create the operation field.

Total gastrectomy with adequate regional lymph node dissection (D1 and stations No. 7, 8a, 9, and 11p) was performed according to the following procedures. Briefly, after the left and right gastroepiploic vessels were divided to dissect the perigastric and infrapyloric lymph nodes, the duodenum was transected with a 45 $\mathrm{mm}$ endoscopic linear stapler (ETS-Flex45; Ethicon Endo-Surgery Japan, Tokyo, Japan). The dissection was then advanced to remove the lymph nodes along the branches of the celiac artery (stations No. 7, 8a, 9 , and 11p). The lesser omentum was divided to the esophago-cardiac junction, preserving the hepatic branch of the vagus nerve, to expose the right side of the esophagus. The splenic artery was exposed to the hilus of the spleen while the posterior gastric artery was divided at its origin. Short gastric vessels were divided along the spleen and the fundic portion of the stomach was mobilized to expose the esophagus. Finally, the phreno-esophageal membrane and the anterior and posterior vagal trunks were divided to expose the abdominal esophagus completely.

After preparation of the esophagus, a jejunal loop about $20 \mathrm{~cm}$ distal to the ligament of Treitz was marked with a dye (pyoktanin blue) for subsequent Roux-en-Y reconstruction. A 5-cm midline skin incision was made in the upper abdomen, extending the wound, through which the liver retractor was introduced. The abdominal cavity was entered and an Alexis Wound Retractor S (Applied Medical, Rancho Santa Margarita, CA, USA) was fitted to the wound to prevent infection. The marked jejunal loop was pulled out through the laparotomy and transected, then side-to-side jejunojejunos-

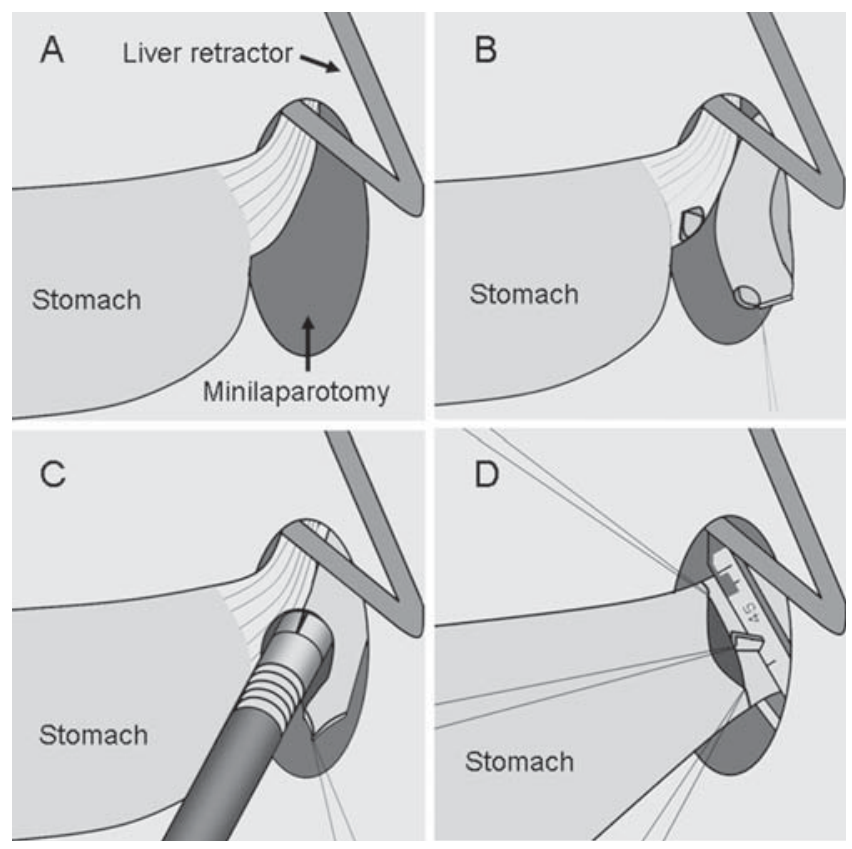

Fig. 2A-D. Schematic outline of linear-stapled esophagojejunostomy through a minilaparotomy. A Stomach is pulled out from the minilaparotomy. B Holes are made in the left side of the esophagus and the end of the jejunal limb. C An endoscopic linear stapler is applied through the two holes to create an anastomosis. D The access opening is closed with a stapler inserted through the left lower trocar

tomy (Y-anastomosis) was performed using the ETS-Flex45 to create a 50-cm Roux-en-Y limb. The entry hole of the stapler was closed by interrupted 3-0 PDS (Ethicon, San Angelo, TX, USA) hand sutures. After the end of the Roux-en-Y limb was marked by a 3-0 PDS suture, the Y-anastomosis was placed back into the abdominal cavity. The entire stomach was taken out from the wound and a small hole was made on the left side of the esophagus just proximal to the esophagocardiac junction (Fig. 2A,B). The Roux-en-Y limb was then brought up via the antecolic route and a small hole was made at its end on the anti-mesenteric side (Fig. 2B). The ETS-Flex45 was applied through the minilaparotomy with one jaw in each small hole to make a side-to-side anastomosis between the esophagus and the jejunal limb (Figs. 2C, 3A). Before the closure of the entry hole with the stapler, it was roughly closed with three 3-0 PDS supporting sutures. To complete the closure and transect the esophagus to finish the gastrectomy concurrently, the ETS-Flex45 was applied through the left lower port so that all three supporting sutures were included in the resected specimen (Fig. 3B). The closure was monitored under direct vision through the laparotomy (Fig. 2D). In some cases, further application was necessary to complete the esophageal transection. 


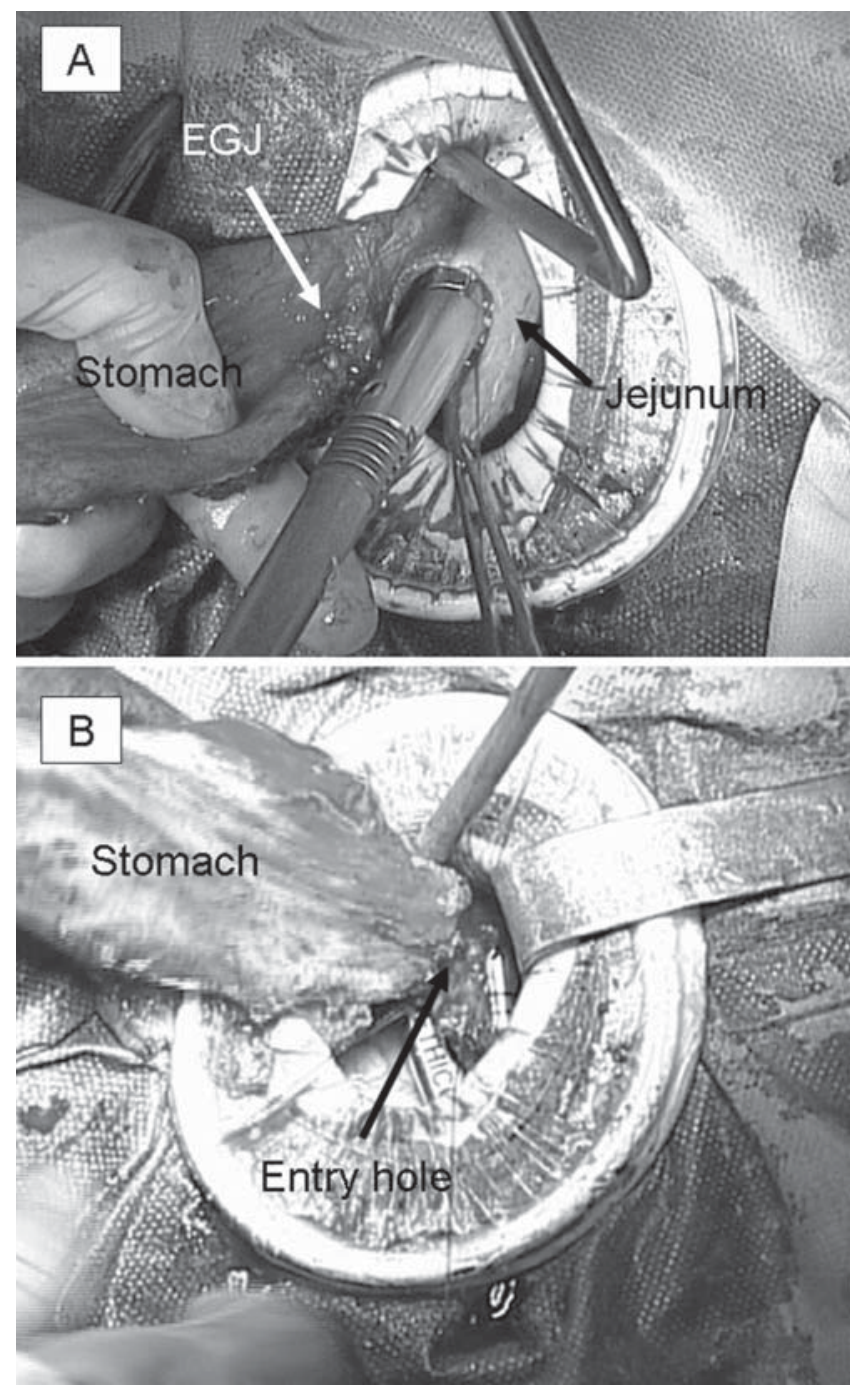

Fig. 3. A An endoscopic linear stapler is operated through the minilaparotomy to make an esophagojejunal anastomosis. $E G J$, esophagogastric junction. B Closure of the entry hole is monitored through the minilaparotomy

To confirm the tightness of the anastomosis, an air leakage test was performed by delivering $50 \mathrm{ml}$ of air through a nasogastric tube to the esophagojejunostomy (Fig. 4). A flat-type suction drain was inserted from the upper right port and placed behind the esophagojejunal anastomosis to the left subphrenic space. The operation was completed by closure of all trocar wounds.

\section{Results}

The mean operative time for the seven patients was $283 \mathrm{~min}$ (range, 227 to $322 \mathrm{~min}$ ), and the estimated blood loss was $203 \mathrm{~g}$ (range, 30 to $420 \mathrm{~g}$ ). All Roux-en$Y$ reconstructions were performed through a 5-cm laparotomy (Fig. 5). We did not experience any cases that

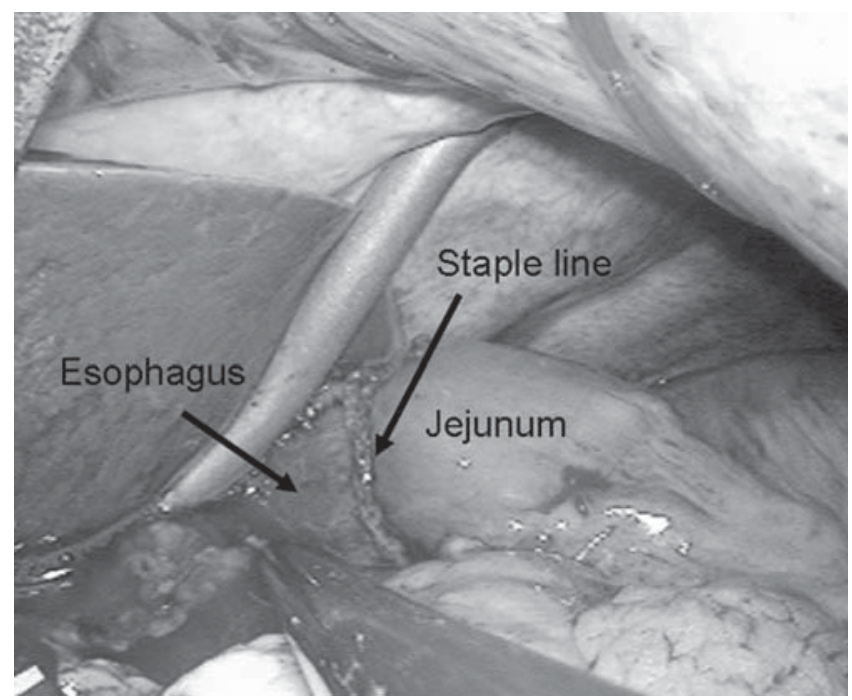

Fig. 4. Laparoscopic view of the anastomosis. A staple line for closing the common entry hole is seen

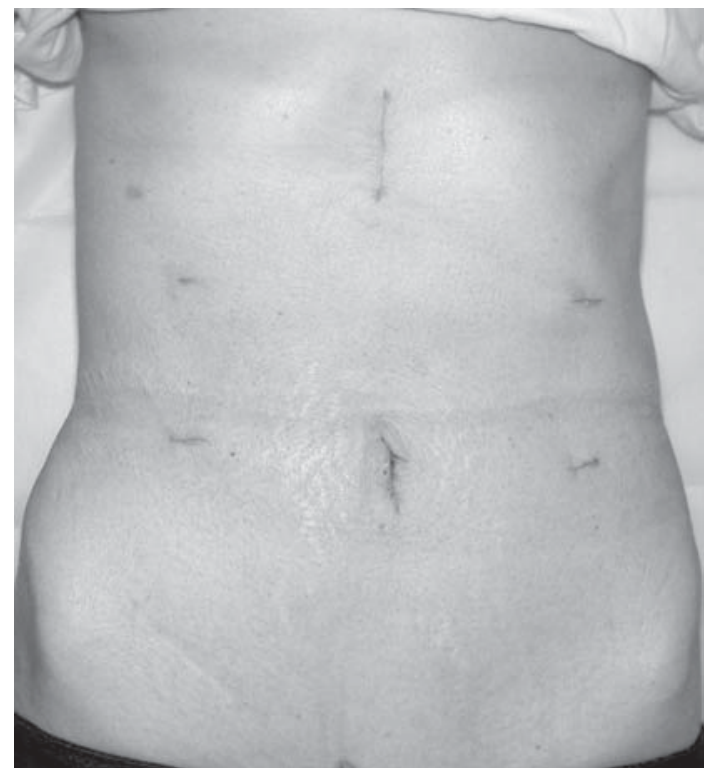

Fig. 5. Postoperative view of surgical wounds 3 months after laparoscopic total gastrectomy (LTG)

required extension of the wound to complete the reconstruction. No intraoperative complications were experienced. All operations were curative and the median number of retrieved lymph nodes was 54.3 (range, 17 to 85 ).

One patient developed intraabdominal abscess, which resolved following treatment with antibiotics. There were no other postoperative complications. Postoperative fluorography demonstrated neither stenosis nor leakage of the anastomosis (Fig. 6). All patients resumed intake of clear liquid on the third postoperative day and 


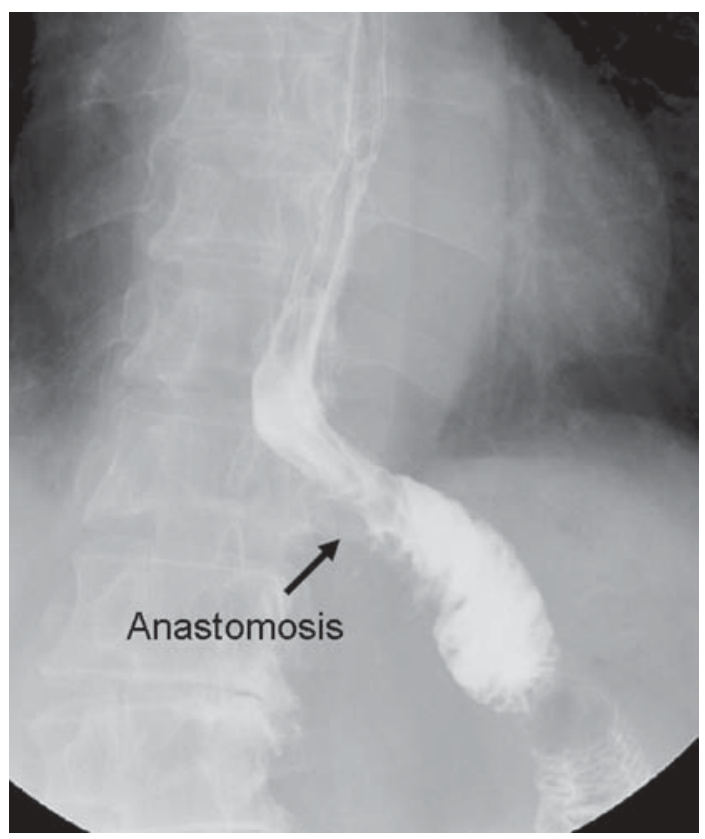

Fig. 6. Postoperative fluorography demonstrates normal passage of oral hydrosoluble contrast without leakage or stenosis of the anastomosis

ingested food on the following day. The median postoperative hospital stay was 10 days (range, 9 to 14 days). There was no mortality.

\section{Discussion}

Although the long-term outcome of laparoscopic surgery for early gastric cancer has not been elucidated, LDG is spreading as one of the standard surgical treatments in several countries [1-3]. In LDG, most surgeons prefer to perform reconstructions through an extended wound [4-6], because the surgical field required for gastrojejunostomy or gastroduodenostomy is easily accessible through a small upper abdominal incision. However, it is often difficult to complete Roux-en-Y reconstruction after LTG through a minilaparotomy.

In open total gastrectomy, esophagojejunal anastomosis is mostly performed using circular stapling devices. According to previous reports, esophagojejunostomy in LTGs has also been performed using circular staplers in most cases $[3,7]$. The extracorporeal use of circular staplers is preferred compared with their intracorporeal use, because it is hard to operate circular staplers under the limited laparoscopic view. However, even using an extracorporeal method, there are still potential problems in completing esophagojejunostomy with a circular stapling device. Insertion of an anvil into the esophagus is sometimes problematic, because the esophagus is shortened after it is transected, and may lead to disruption of the esophageal wall. Because the operation field is deeper than that in distal gastrectomy, it is also difficult to maintain adequate tension and to observe the anastomosis under direct view while a stapling device is applied. As a result, extension of the incision is often required to complete the reconstruction safely.

To avoid problems with circular staplers, linearstapled esophagojejunostomy has also been reported as an intracorporeal reconstruction method after LTG [8, 9]. However, even in these procedures, it can be troublesome to close the access opening securely after a common hole is generated by the first application of a linear stapler. Some have reported closure with further application of linear staplers $[9,10]$. In this method, linear stapling to achieve complete closure of the entry hole is not easy under the limited laparoscopic view and may lead to stenosis of the anastomosis. To prevent possible stenosis, others have reported closure with hand sutures, which takes longer to perform and requires a higher degree of surgical skill [8]. Considering the reports comparing hand-sutured and stapled esophagojejunostomies, completion of esophagojejunostomy by hand sutures may lead to an increased rate of leakage [11].

To overcome these potential problems and securely perform a Roux-en-Y reconstruction through a small epigastric wound after LTG, we made some modifications to a previously reported linear-stapled esophagojejunostomy method [12]. First, for easier handling of the esophagus, the first application of the linear stapler to create the esophagojejunal anastomosis was performed before transection of the esophagus. Because we are not able to examine the oral margin during the operation, we generally exclude patients with tumor invading esophagus from this method. Second, the jejunal limb was anastomosed to the left side of the esophagus, not to the right side, which has been used conventionally. Compared to the right side, more free space, sufficient to operate the stapling device was made available, even in patients with a large lateral segment of the liver. Third, the stapler for the closure of the common hole was operated through the left lower trocar, not via the minilaparotomy. With this technique, the closure of the access opening, which is the most important last step of the anastomosis, was performed easily and was monitored directly through the minilaparotomy. Although we do not have any experience in very obese patients, we expect that, in such patients, the first stapling would be more difficult and extension of the laparotomy may be necessary for safety in some cases.

In all seven patients, we successfully performed Rouxen-Y reconstruction through a 5-cm upper abdominal incision and experienced neither anastomotic leakage 
nor stenosis. We believe our procedure will facilitate the acceptance of LTG as a surgical option for patients with early proximal gastric cancers.

\section{References}

1. Huscher C, Mingoli A, Sgarzini G, Sansonetti A, Di Paola M, Recher A, et al. Laparoscopic versus open subtotal gastrectomy for distal gastric cancer: 5-year results of a randomized prospective trial. Ann Surg 2005;241:232-7.

2. Kitano S, Shiraishi N, Uyama I, Sugihara K, Tanigawa N. A multicenter study on oncologic outcome of laparoscopic gastrectomy for early cancer in Japan. Ann Surg 2007;245:68-72.

3. Dulucq J, Wintringer P, Perissat J, Mahajna A. Completely laparoscopic total and partial gastrectomy for benign and malignant diseases: a single institute's prospective analysis. J Am Coll Surg 2005;200:191-7.

4. Adachi Y, Shiraishi N, Shiromizu A, Bandoh T, Aramaki M, Kitano S. Laparoscopy-assisted Billroth I gastrectomy compared with conventional open gastrectomy. Arch Surg 2000;135: 806-10.

5. Mochiki E, Kamiyama Y, Aihara R, Nakabayashi T, Asao T, Kuwano H. Laparoscopic assisted distal gastrectomy for early gastric cancer: 5 years' experience. Surgery 2005;137:317-22.
6. Takaori K, Nomura E, Mabuchi H, Lee S, Agui T, Miyamoto Y, et al. A secure technique of intracorporeal Roux-Y reconstruction after laparoscopic distal gastrectomy. Am J Surg 2005;189: 178-83.

7. Usui $S$, Yoshida $T$, Ito $K$, Hiranuma $S$, Kudo $S$, Iwai $T$. Laparoscopy-assisted total gastrectomy for early gastric cancer: comparison with conventional open total gastrectomy. Surg Laparosc Endosc Percutan Tech 2005;15:309-14.

8. Huscher C, Mingoli A, Sgarzini G, Sansonetti A, Lirici M, Napolitano C, et al. Videolaparoscopic total and subtotal gastrectomy with extended lymph node dissection for gastric cancer. Am J Surg 2004;188:728-35.

9. Uyama I, Sugioka A, Fujita J, Komori Y, Matsui H, Hasumi A. Laparoscopic total gastrectomy with distal pancreatosplenectomy and D2 lymphadenectomy for advanced gastric cancer. Gastric Cancer 1999;2:230-4.

10. Tanimura S, Higashino M, Fukunaga Y, Kishida S, Ogata A, Fujiwara Y, et al. Laparoscopic gastrectomy with regional lymph node dissection for upper gastric cancer. Br J Surg 2007;94: 204-7.

11. Nomura S, Sasako M, Katai H, Sano T, Maruyama K. Decreasing complication rates with stapled esophagojejunostomy following a learning curve. Gastric Cancer 2000;3:97-101.

12. Matsui H, Uyama I, Sugioka A, Fujita J, Komori Y, Ochiai M, et al. Linear stapling forms improved anastomoses during esophagojejunostomy after a total gastrectomy. Am J Surg 2002;184: $58-60$. 